\title{
SUPERCONDUCTING CHARACTERISTICS OF THE PENSON-KOLB MODEL
}

\author{
W.R. CZart and S. RobaszKIEWICZ \\ Institute of Physics, Adam Mickiewicz University, Umultowska 85, 61-614 Poznań
}

We study superconducting properties of the Penson-Kolb model, i.e. the tight-binding model with the pair-hopping (intersite charge exchange) interaction $J$. The evolution of the critical fields, the coherence length, the Ginzburg ratio, and the London penetration depth with particle concentration $n$ and pairing strength are determined. The results are compared with those found earlier for the attractive Hubbard model.

PACS numbers: $74.20 .-\mathrm{z}, 71.28 .+\mathrm{d}, 74.25 . \mathrm{Ha}$

\section{General formulation}

The Penson-Kolb (PK) model is one of the conceptually simplest phenomenological models for studying superconductivity in systems with short-range, almost unretarded pairing $[1,2]$. It includes a nonlocal pairing mechanism (the pair hopping term $J$ ) that is distinct from the on-site interaction in the attractive Hubbard $\mathrm{AH})$ model and that is the driving force of pair formation and also of their condensation. Thus, the superconducting properties can be essentially different in these two models [2]. In the paper we focus on the PK model with arbitrary particle concentration and discuss its superfluid characteristics which have not been considered up to now. In the analysis we have used a linear response theory $[3,4]$ and the electromagnetic kernel has been evaluated within HFA-RPA scheme. The model Hamiltonian has the following form:

$$
\begin{aligned}
H= & -t \sum_{\langle i j\rangle \sigma}\left[\exp \left(\mathrm{i} \Phi_{i j}\right) c_{i \sigma}^{+} c_{j \sigma}+\text { h.c. }\right] \\
& -\frac{1}{2} J \sum_{\langle i j\rangle}\left[\exp \left(2 \mathrm{i} \Phi_{i j}\right) c_{i \uparrow}^{+} c_{i \downarrow}^{+} c_{j \downarrow} c_{j \uparrow}+\text { h.c. }\right],
\end{aligned}
$$

where $t$ is the single electron hopping integral, $J$ is the pair hopping (intersite charge exchange) interaction, the limit $\langle i j\rangle$ restricts the sum to nearest neighbors (nn). The Peierls factors in (1) account for the coupling of electrons to the magnetic field via its vector potential $\boldsymbol{A}(r): \Phi_{i j}=(-e / \hbar c) \int_{R_{i}}^{R_{j}} \mathrm{~d} \boldsymbol{r} \boldsymbol{A}(\boldsymbol{r})$, and $e$ is the electron charge. 
From the linear response theory $[3,4]$ the expectation value of the Fourier transform of the total current operator is

$$
J_{\alpha}(\boldsymbol{q}, \omega)=N \frac{c}{4 \pi} \sum_{\beta}\left[\delta_{\alpha \beta} K_{\alpha}^{\mathrm{dia}}+K_{\alpha \beta}^{\mathrm{para}}(\boldsymbol{q}, \omega)\right] A_{\beta}(\boldsymbol{q}, \omega) .
$$

The diamagnetic part of kernel evaluated within HFA has the following form:

$$
K_{\alpha}^{\text {dia }}=\frac{8 \pi e^{2}|t|}{\hbar^{2} c^{2} a} \frac{1}{N} \sum_{k}\left[1-\frac{\bar{\epsilon}_{k}}{E_{k}} \tanh \left(\frac{\beta E_{k}}{2}\right)\right] \cos \left(k_{\alpha}\right)-\frac{32 \pi e^{2}}{\hbar^{2} c^{2} a} \frac{J_{0}}{z} x_{s}^{2},
$$

where $\bar{\epsilon}_{k}=\epsilon_{k}-\mu, E_{k}=\sqrt{\bar{\epsilon}_{k}^{2}+\Delta^{2}}, \Delta=J_{0} x_{s}, J_{0}=z J, \epsilon_{k}=-2 \tilde{t} \gamma_{k}$, $\tilde{t}=t+2 p J / z, \mu$ is the chemical potential, $a$ is the lattice constant, $\gamma_{k}=\sum_{\alpha} \cos k_{\alpha}$, $\alpha=x, y, \ldots$, and $z$ is the number of nn. The superconducting order parameter $x_{s}=(1 / N) \sum_{i}\left\langle c_{i \downarrow} c_{i \uparrow}\right\rangle$, the Fock term $p=(1 / 4 N) \sum_{k \sigma} \gamma_{k}\left\langle c_{k \sigma}^{+} c_{k \sigma}\right\rangle$ and $\mu$ are determined by the equations $\partial F_{\mathrm{S}} / \partial x_{s}=0, \partial F_{\mathrm{S}} / \partial p=0, \partial F_{\mathrm{S}} / \partial \mu=0$, with $F_{\mathrm{S}}$ being the free energy of the superconducting (S) phase

$$
\frac{F_{\mathrm{S}}}{N}=\mu(n-1)+\frac{4}{z} J p^{2}+J_{0} x_{s}^{2}-\frac{2}{\beta N} \sum_{k} \ln \left[2 \cosh \left(\frac{\beta E_{k}}{2}\right)\right] .
$$

In the static limit and $q \rightarrow 0$ for the transverse part of the paramagnetic kernel we obtain

$$
K_{x x}^{\text {para }}(\omega=0)=\frac{8 \pi e^{2} t^{2}}{\hbar^{2} c^{2} a} \frac{1}{N} \sum_{\boldsymbol{k}} \frac{\sin ^{2} k_{x}}{k_{\mathrm{B}} T \cosh ^{2}\left(\beta E_{k} / 2\right)} .
$$

In the local approximation (London limit) the magnetic penetration depth is determined in terms of the transverse part of the total kernel as $\lambda=\left[-K_{x}^{\text {dia }}-\right.$ $\left.K_{x x}^{\text {para }}(\omega=0)\right]^{-1 / 2}$. Using the value of $\lambda$ and the difference of the free energy between the normal $(\mathrm{N})$ and $\mathrm{S}$ phases one is able to determine the thermodynamic critical field $H_{\mathrm{c}}$ and the Ginzburg-London correlation length $\xi_{\mathrm{GL}}$ as $H_{\mathrm{c}}^{2}(T) / 8 \pi=$ $\left[F_{\mathrm{N}}(T)-F_{\mathrm{S}}(T)\right] / N a^{3}, \xi_{\mathrm{GL}}=\Phi_{0} / 2 \pi \sqrt{2} \lambda H_{\mathrm{c}}$, where $\Phi_{0}=h c / 2 e$, and to obtain the estimations for the critical fields $H_{\mathrm{c} 1} \simeq(\ln \kappa / \kappa) H_{\mathrm{c}}$ and $H_{\mathrm{c} 2}=\Phi_{0} /\left(2 \pi \xi_{\mathrm{GL}}^{2}\right)$, where $\kappa=\lambda / \xi_{\mathrm{GL}}$.

\section{Results of numerical solutions and discussion}

Examples of the evolution of the penetration depth (its inverse square value $\left.1 / \lambda^{2}\right)$, the critical field $H_{\mathrm{c}}$, the coherence length $\xi_{\mathrm{GL}}$ and the Ginzburg ratio $\kappa=\lambda / \xi_{\mathrm{GL}}$ with $n$ are shown in Fig. 1, for $d=2 \mathrm{SQ}$ lattice and a fixed value of $J_{0} / B$, whereas Fig. 2 shows the plots of $H_{\mathrm{c}}^{2}$ (for $d=2 \mathrm{SQ}$ lattice), $1 / \lambda^{2}$ and $\xi_{\mathrm{GL}}$ (for $d=2 \mathrm{SQ}$ and $d=3 \mathrm{SC}$ lattices) as a function of $J_{0} / B$ for $n=1$. As $J$ increases the $1 / \lambda^{2}$ evolves smoothly between the limit of weakly interacting single-particle carriers (with $\lambda^{-2}$ being proportional to the bandwidth $B$ ) and that of tightly bound pairs (with $\lambda^{-2} \sim J$ ). Notice the increase in $\lambda^{-2}$ as a function of $J_{0}$ (cf. Fig. 2), i.e. the behavior being qualitatively different than that found for the $\mathrm{AH}$ model, where $\lambda^{-2}$ continuously decreases with increasing $|U| / B[4,5]$. One also finds that in the low density limit $\lambda^{-2} \sim n$ for arbitrary $J_{0} / B$ (cf. Fig. 1), whereas for $J_{0} / B \gg 1: \lambda^{-2} \sim n(2-n)$ for any $n$. With increasing $J$ the $H_{\text {c }}^{2}$ 


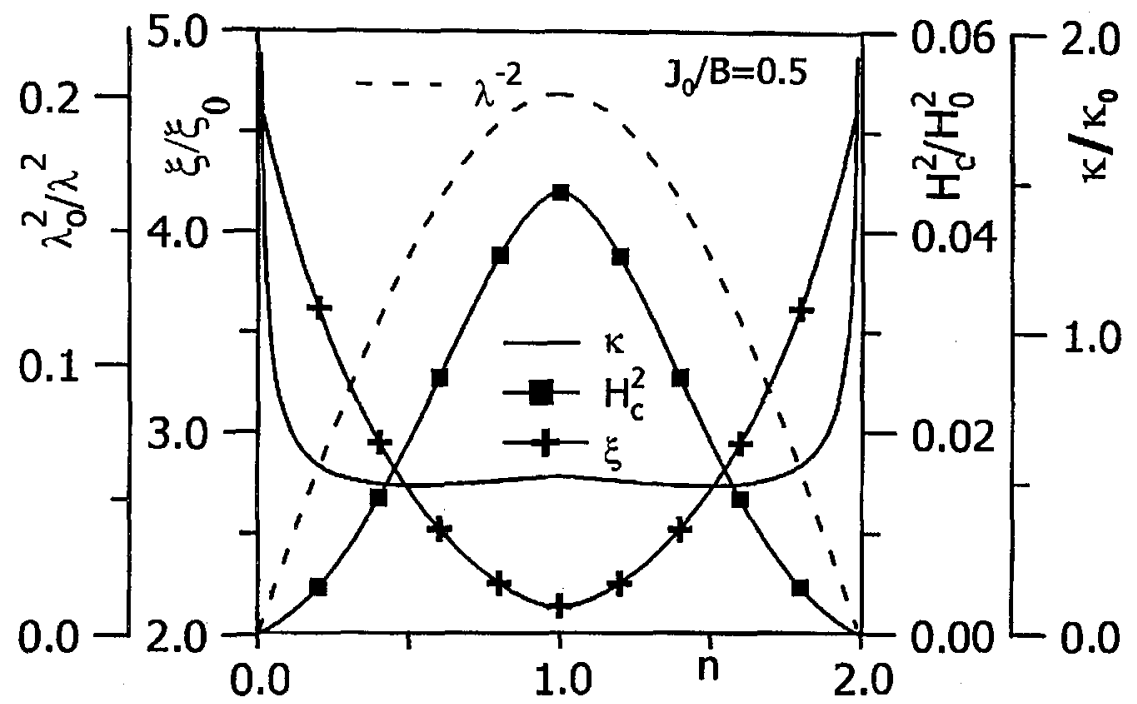

Fig. 1. Concentration dependences of $1 / \lambda^{2}, H_{c}^{2}, \xi_{\mathrm{GL}}$, and $\kappa=\lambda / \xi_{\mathrm{GL}}$ at $T=0$ for $d=2$ square (SQ) lattice and $J_{0} / B=0.5(B=2 z t) . \lambda_{0}=(\hbar c / e) \sqrt{a^{d-2} / 4 \pi B}, H_{0}^{2}=4 \pi B / a^{d}$, $\xi_{0}=a / 2 \sqrt{2}, \kappa_{0}=(\hbar c / e) \sqrt{2 / \pi B a^{4-d}}$.

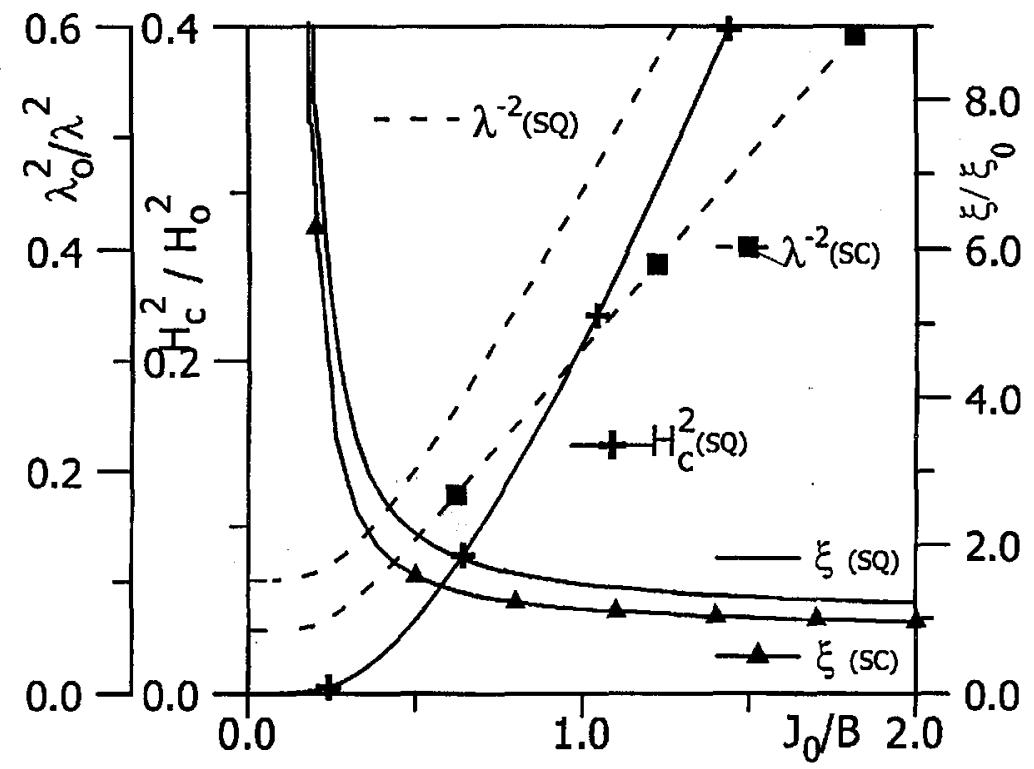

Fig. 2. The plots of $H_{\mathrm{c}}^{2}$, for $d=2 \mathrm{SQ}$ lattice, and $1 / \lambda^{2}, \xi_{\mathrm{GL}}$, for $d=2$ (SQ) and $d=3$ (SC) lattices, as a function of $J_{0} / B$ for $n=1$. The units as in Fig. 1.

increases exponentially at small $J$ and becomes proportional to $J_{0}$ in the strong coupling (Fig. 2).

The $\xi_{\mathrm{GL}}$ rapidly decreases at small $J\left(\xi_{\mathrm{GL}} \sim \exp \left(-2 J_{0} / B\right)\right.$, for $\left.J_{0} \ll B\right)$ and tends to a constant value $\xi_{\infty}=a / \sqrt{2 z}$, the same for all $n$, at large $J$. Note 
a substantial variation of $\xi_{\mathrm{GL}}$ with $n$ in the weak-to-intermediate coupling regime (cf. Fig. 2), where $\xi_{\text {GL }}$ attains the minimal value at half-filling. This feature is largely due to the strong $n$ dependence of $H_{\mathrm{c}}^{2}$, which in weak coupling is proportional to $\Delta^{2} D(\bar{\mu})$, where $D(\varepsilon)$ is the density of states (DOS) function.

As it follows the analysis of limiting cases the increase in the Ginzburg ratio $\kappa=\lambda / \xi_{\mathrm{GL}}$ with $J$ is exponential in the weak coupling limit $\left[\kappa \sim \exp \left(-B / J_{0}\right)\right]$, whereas in the opposite limit $\kappa$ becomes proportional to $\sqrt{1 / J}$. A crossover between these two types of behavior takes place for intermediate values of $J$ $\left(1<J_{0} / B<2\right)$. In definite limits one finds universal $\kappa$ vs. $n$ dependences: (i) $\kappa \sim[n(2-n)]^{-1 / 2}$, for $J_{0} / B \gg 1$ (arbitrary $n$, any lattice) and (ii) $\kappa \sim 1 / \sqrt{n}$, for $n \ll 1$ (arbitrary $J_{0} / B$, SQ lattice), being analogous to those obtained for the attractive Hubbard model [4]. Beyond these limits $\kappa(n)$ is not universal and strongly depends on the details of $D(\varepsilon)$. In particular, the local maximum of $\kappa$ at $n=1$ seen in Fig. 1 results from the van Hove singularity in $D(\varepsilon)$ for SQ lattice.

\section{Final comment}

Let us stress that due to the nonlocal pairing mechanism (intersite charge exchange) the dynamics of electron pairs in the PK model is qualitatively different from that in the attractive Hubbard model [2]. It results in different thermodynamic and electrodynamic properties of both models, especially in the strong coupling limit.

In the latter model with increasing $|U|$ the $T_{\mathrm{c}}$ and $H_{\mathrm{c}}^{2}$ increase exponentially for small $|U|$, then they go through a round maximum and they decrease as $t^{2} /|U|$ for large coupling $[4,5]$. On the contrary, in the PK model there is no maximum of $H_{\mathrm{c}}^{2}$ and $T_{\mathrm{c}}$ at intermediate $J / t$ and both these quantities increase linearly with $J$ for large coupling (cf. Fig. 2 and Ref. [2]). Also the behavior of the penetration depth $\lambda$ is different. In particular, for $t \rightarrow 0: \lambda$ decreases with $J$ in the PK model $\left(\lambda^{2} \sim 1 / J\right)$, while it increases with $|U|$ in the AH model $\left(\lambda^{2} \sim|U| / t^{2}[4]\right)$.

In this report we have concentrated on the electromagnetic properties of the model at $T=0$. A detailed study of the finite temperature behavior, taking into account the effects of phase fluctuations in $d=2$ system (within the framework of Kosterlitz-Thouless scenario) and providing a rigorous solution in the case of $d=\infty$ system will be given elsewhere.

We thank B. Bułka and R. Micnas for helpful discussion. This work is supported through the Committee for Scientific Research grants No. 2 P03B 03516 and 2 P03B 05709.

\section{References}

[1] K.A. Penson, M. Kolb, Phys. Rev. B 33, 1663 (1986).

[2] S. Robaszkiewicz, B. Bułka, Phys. Rev. B 59, 6430 (1999) and references therein.

[3] D.J. Scalapino, S.R. White, S. Zhang, Phys. Rev. B 47, 7995 (1993).

[4] W.R. Czart, T. Kostyrko, S. Robaszkiewicz, Physica C 272, 51 (1996); B. Bułka, S. Robaszkiewicz, Phys. Rev. B 54, 13138 (1996).

[5] J.M. Singer, T. Schneider, M. Pedersen, Eur. Phys. J. B 2, 17 (1998); M. Bak, R. Micnas, J. Phys., Condens. Matter 10, 9029 (1998). 